\title{
Anti-dsDNA negative and anti-Ro positive lupus nephritis: a report of a rare case
}

\author{
D. Jain, H.K. Aggarwal, V. Kaverappa, S. Dhayia, P. Jain, S. Yadav \\ Department of Medicine, Pt. B.D. Sharma University of Health Sciences, India
}

\begin{abstract}
SUMMARY
Systemic lupus erythematosus (SLE) is a multisystem autoimmune disease, characterized by an autoantibody response to various nuclear and cytoplasmic antigens. Renal disease in SLE occurs in 40-75\% of patients, most often within five years of onset of disease, and is one of the strongest predictors of a poor outcome. A hallmark of glomerular involvement in lupus nephritis is the presence of autoantibodies against double-stranded DNA (dsDNA). Its level usually correlates with disease activity. Our patient presented with a rash resembling malar rash and features of nephrotic syndrome. On investigating, patient was found to have pancytopenia, raised erythrocyte sedimentation rate and depressed serum C3 levels with positivity of antinuclear antibodies and antiRo antibodies. However, most of the markers of lupus nephritis including anti dsDNA antibody were negative. Renal biopsy showed features of lupus nephritis (class-IV). Differential item functioning studies showed a full house immunoflourescence staining pattern characteristic of lupus nephritis. Association of Anti-Ro antibody alone with lupus nephritis is less known in literature. Negativity of anti-dsDNA antibody, which is usually considered to be diagnostic of lupus nephritis, poses a diagnostic dilemma short of renal biopsy. Till date only very few cases of non-drug induced lupus nephritis with negative dsDNA antibodies have been reported. In this report we wish to highlight a case of lupus nephritis which was negative for its specific anti dsDNA antibodies and with possible role of anti-Ro antibodies in the pathogenesis of lupus nephritis although the underlying mechanism is incompletely understood.
\end{abstract}

Key words: Lupus nephritis, Systemic lupus erythematosus.

Reumatismo, 2013; 65 (6): 302-306

\section{INTRODUCTION}

ystemic lupus erythematosus (SLE) is $\checkmark$ a multisystem disease, autoimmune in origin, characterized by an autoantibody response to various nuclear and cytoplasmic antigens. Diagnosis of SLE can be made by fulfilling the modified criteria of American criteria of rheumatology. Antinuclear antibodies (ANA) positivity is usually considered as hallmark of SLE being positive in more than $95 \%$ of patients (1). Almost about half of the patients develop features of renal involvement during course of disease; lupus nephritis being the most severe form. Lupus nephritis has been associated with presence of many specific antibodies such as dsDNA which correlate with the disease activity. SLE has also been associated with other autoimmune disease such as Sjögren's syndrome and autoimmune thyroid disorders. In this report we highlight the case of a hypothyroid fe- male presenting with lupus nephritis who was negative for anti dsDNA antibodies. However; her sera was positive for anti Ro antibodies with no evidence suggestive of primary Sjögren's syndrome.

\section{CASE REPORT}

A 40-year female, housewife, known hypothyroid on regular thyroxine replacement for past ten years, presented with complaints of swelling around eyes and pedal edema for 4 months. It started initially as mild periorbital puffiness usually in morning which later persisted throughout the day, gradually progressing to swelling bilateral feet upto ankles. She also complained of reddish discoloration over nose and malar prominence which was not aggravated by exposure to sunlight. Further enquiry revealed that she was alsoexperiencing easy fatigability for last 6 


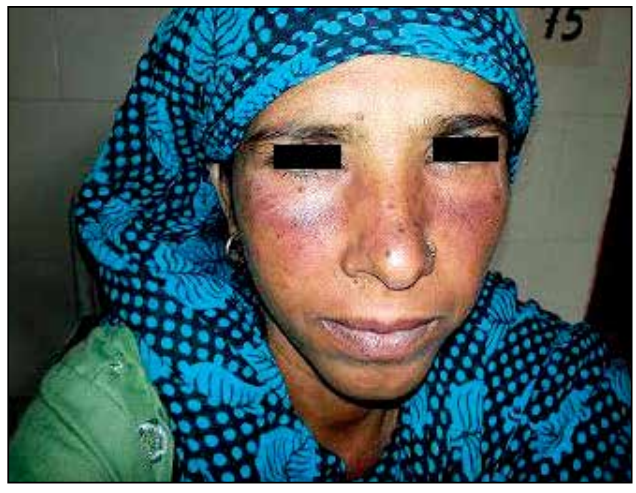

Figure 1 - An ill-defined erythematous plaque over bilateral malar eminence suggestive of malar rash.

months. She had no history of fever or sore throat,oral ulcers, joint pains, decreased urine output, or blood in urine.History of dryness of mouth or eyes was conspicuously absent. Apart from intake of thyroxine, her past history including drug history and obstetric history, and family history was unremarkable.

On physical examination, she was hemodynamically stable with blood pressure of $130 / 90 \mathrm{mmHg}$ and pulse rate of $80 \mathrm{bpm}$. Pallor was present, so was pedal edema which was pitting in nature. She had an illdefined erythematous plaque over bilateral malar eminence which was suggestive of malar rash (Figure 1). Her cardiovascular, respiratory, abdominal and central nervous system examination was essentially normal. Her complete hemogram revealed a picture of pancytopenia with hemoglobin of $7.5 \mathrm{gm} \%$; total leukocyte count $3 \times 10^{9} / \mathrm{L}$, and platelet count of $100 \times 10^{9} / \mathrm{L}$ with dimorphic picture. Her renal functions were normal with blood urea nitrogen of $10 \mathrm{mg} / \mathrm{dL}$ and serum creatinine of $0.8 \mathrm{mg} / \mathrm{dL}$. Liver function tests, blood sugar and serum electrolytes levels were well within normal physiological limits. Her serum triglycerides and cholesterol were raised. Hypoproteinemia was present with serum protein level of $5.9 \mathrm{gm} / \mathrm{dL}$ and albumin to globulin ratio of 0.9 . Thyroid function tests revealed normal serum free T3, T4 and TSH levels with a raised anti TPO antibody titers to the level of $650 \mathrm{IU} /$ $\mathrm{mL}$. Urine complete examination revealed
$3+$ proteinurea by dipstick, numerous dysmorphic red blood cells (RBCs) and many waxy, granular and RBC casts. Her proteinuria was quantified to be $3.3 \mathrm{~g} /$ day after $24 \mathrm{~h}$ collection of urine. Serology for HIV I and II, hepatitis B and hepatitis C were negative. Although her C-reactive protein (CRP) levels, rheumatoid factor, and ASO titers were negative erythrocyte sedimentation rate (ESR) was raised to $70 \mathrm{~mm}$ in the first hour. Hypocomplimentemia was characteristically present with reduced levels of C3 (0.40/L). ANA was positive by ELISA, while immunofluorescence showed speckled pattern at titers of 1:80. Her serology was positive for anti SS-A(Ro) antibodies $(44.2 \mathrm{U} / \mathrm{mL})$, however; anti-dsDNA (by Farr assay and ELISA), anti-smith antibody, anti-U1RNP, anti-centromere antibody, and anti SS$\mathrm{B}(\mathrm{La})$ antibody were negative.

Her ultrasonography revealed right kidney of $12.5 \times 4.3 \mathrm{~cm}$, left kidney $11.9 \times 4.3$ $\mathrm{cm}$ with bilaterally increased echogenicity. Renal biopsy was performed under ultrasound guidance which on microscopic examination showed 10 glomeruli, among which one was globally sclerosed. Remainder showed variable mesangial proliferation and matrix expansion. Patchy, segmental endocapillary cellularity was seen

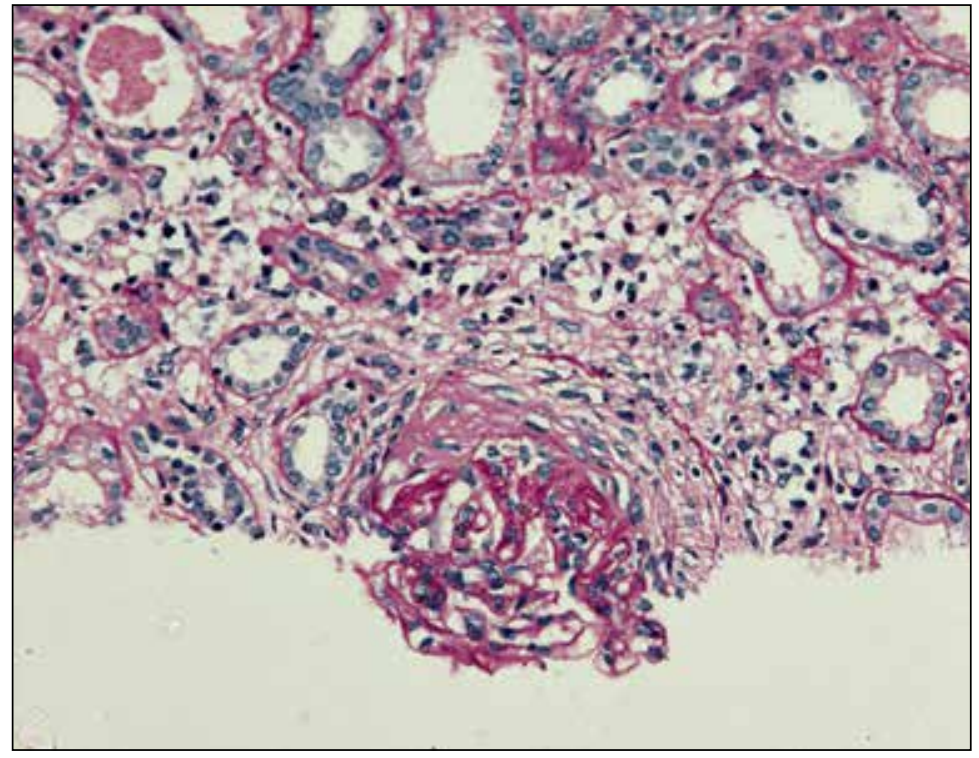

Figure 2 - Renal biopsy consistent with lupus nephritis. 
including mononuclear cells as well as polymorphs. One glomerulus exhibited a segmental scar with an overlying fibrocellular crescent (Figure 2). Differential item functioning studies on the biopsy specimen showed a full house immunostaining pattern with capillary wall and mesangial deposits of immunoglobulin (Ig) G, IgA, C3 and $\mathrm{C} 1 \mathrm{q}$ and less intense IgM. These findings were consistent with lupus nephritis (Class IV). Patient was further investigated for Sjögren's syndrome with Schirmer test and labial biopsy for minor salivary glands; which turned out negative. Sjögren's syndrome was excluded as none of the criteria were fulfilled apart from anti-Ro positivity. Eventually, patient was diagnosed as a case of SLE with lupus nephritis (class-IV) fulfilling at least three clinical, two immunological and proven renal biopsy finding as per SLICC criteria (2).

The patient was given a pulse of intravenous methyl prednisone $1 \mathrm{~g}$ per day for three days, followed by maintenance therapy of oral prednisone at $1 \mathrm{mg} / \mathrm{kg}$ body weight. Her blood counts were monitored serially until it recovered, and aggressive immunosuppression was started with intravenous cyclophosphamide as pulse therapy (500 mg every 2 weeks $\times 6$ cycles) with regular monitoring of complete blood counts. At end of 3 months her proteinuria decreased to $<200 \mathrm{mg} /$ day. She has been maintained on azathioprine (50 mg bid) since 2 months post-pulse therapy. Her proteinurea is being monitored on follow up visits which is persistently below $100 \mathrm{mg} /$ day.

\section{DISCUSSION}

Systemic lupus erythematosus (SLE) is a multisystem autoimmune disorder with a broad spectrum of clinical presentations. The clinical manifestations of SLE are varied with involvement of multiple organ systems during the course of the disease; the damage initially mediated by tissue binding autoantibodies and immune complexes. Various autoantibodies found in SLE include ANA, anti dsDNA, anti Sm, anti RNP, anti Ro, anti La, antiphospholipid antiboby, etc.

Renal disease in SLE occurs in 40-75\% of patients, most often within five years of disease onset, and is one of the strongest predictors of a poor outcome. Anti dsDNA is most commonly associated with nephritis and its level usually correlate with disease activity $(3,4)$. Other nephritogenic antibodies include Anti smith antibody and lupus anticoagulant. Most SLE patients have autoantibodies for 3 or more years before the onset of first symptoms of disease. Anti Ro antibody on the other hand is more commonly associated with sicca syndrome. It also predisposes to subacute cutaneous lupus and neonatal lupus with congenital heart block. Although some studies have shown that anti-Ro/SSA antibodies alone are associated with a higher prevalence of nephritis, others did not find any correlation between Ro/SSA antibodies and nephritis (5). Rather a lower prevalence of central nervous system and renal involvement have been reported (6). Anti-C1q show a significant inverse correlation with levels of $\mathrm{C} 1 \mathrm{q}, \mathrm{C} 3$, and $\mathrm{C} 4$ and decreases in these components of the complement classical pathway are associated with active renal disease. Anti-C1q were detected in $65 \%$ of patients with nephritis, compared with $32 \%$ of those with no renal disease (7).

A number of other autoimmune systemic diseases have been known to occur in association with SLE. In our patient anti Ro antibody positivity also raised the suspicion of associated Sjögren's syndrome but no other criteria was met for its diagnosis as per American-European classification criteria for Sjogren syndrome (8). Therefore a primary diagnosis of SLE with lupus nephritis was made.

SLE disease activity can be evaluated by assessing antibodies to double-stranded DNA (dsDNA), complement (C3, C4), ESR, and CRP levels. Generally, elevated ESR and anti-dsDNA and depressed C3 and $\mathrm{C} 4$ levels are associated with active nephritis, especially focal and diffuse lupus nephritis (4). The negative predictive value of negative anti-dsDNA and anti-C1q anti- 
bodies for active lupus nephritis is $91 \%$ (9). Therefore dsDNA and anti Sm antibodies are always considered as a hallmark for diagnosis of lupus nephritis.

Our patient presented with a rash resembling malar rash and features of nephrotic syndrome. On further investigation patient had pancytopenia, raised ESR with depressed serum C3 levels, however; despite the presence of ANA and anti Ro antibodies, most of the markers of lupus nephritis were negative. Renal biopsy showed full house immunostaining pattern which was a classical picture of lupus nephritis (class-IV). Negativity of anti dsDNA antibody, which is usually considered to be diagnostic of lupus nephritis, poses a diagnostic dilemma short of renal biopsy. Although extremely rare, a few subsets of patients with drug induced lupus nephritis (hydralazine) have been described in the literature to have negative dsDNA and anti-Sm antibodies on serological screening (10). Our patient, however, had no evidence of drug induced lupus nephritis. Short of availability we could not perform anti nucleosome antibodies, however the anti-nucleosome antibody may be a useful marker for diagnosis and activity assessment of anti-dsDNA negative SLE. The levels of anti-nucleosome antibody strongly correlated with the SLEDAI scores, but inversely correlated with serum complement levels in anti-dsDNA negative SLE patients and may be an important factor for renal involvement in this subgroup of patients (11). Till date only very few cases of non drug induced lupus nephritis with negative dsDNA antibodies have been reported. Although multiple hypothesis have been postulated, the exact mechanism underlying this unique finding still remains a mystery. The relatively cationic charged nuclear proteins and various intracellular antigens, derived into circulations after cell death, binding to the negatively charged heparan-sulfated glycosaminoglycan in the glomerular basement membrane. The binding exposes the DNA from the nucleosome that in turn acts as an antigen for anti-dsDNA antibodies (12). The adsorption of circulating anti-dsDNA anti- bodies by the kidney may explain the low levels in the serum. Another explanation could be that the anti-dsDNA could be produced locally against several glomerular targets such as annexin A2, $\alpha$ actinin, alpha enolase, etc. (13). In this report we wish to highlight the association of absence of dsDNA antibodies to lupus nephritis and possible role of anti Ro antibodies in the pathogenesis of lupus nephritis although the underlying mechanism is incompletely understood.

\section{REFERENCES}

1. Tan EM, Feltkamp TEW, Smolen JS, Butcher B, Dawkins R, Fritzler, MJ, et al. Range of antinuclear antibodies in "healthy" individuals. Arthritis Rheum. 1997; 40: 1601-11.

2. Petri M, Orbai AM, Alarcón GS, Gordon C, Merril JTl, Fortin PR, et al. Derivation and validation of the Systemic Lupus International Collaborating Clinics classification criteria for systemic lupus erythematosus. Arthritis Rheum. 2012; 64: 2677-86.

3. Egner W. The use of laboratory tests in the diagnosis of SLE. J Clin Pathol. 2000; 53: 42432.

4. Dooley M. Clinical and laboratory features of lupus nephritis. In: Wallace DJ, Hahn BH, eds. Dubois' lupus erythematosus. 7th ed. Philadelphia, PA: Lippincott Williams \& Wilkins 2007; 1112-30.

5. Wasicek CA, Reichlin M. Clinical and serological differences between systemic lupus erythematosus patients with antibodies to Ro versus patients with antibodies to Ro and La. J Clin Invest. 1982; 69: 835-43.

6. Provost TT, Razzaque A, Maddison PJ, Reichlin M. Antibodies to cytoplasmic antigens in lupus erythematosus: serologic marker for systemic disease. Arthritis Rheum. 1977; 20: 1457-63.

7. Siegert C, Daha M, Westedt ML, van der Voort E, Breedveld F. IgG autoantibodies against $\mathrm{C} 1 \mathrm{q}$ are correlated with nephritis, hypocomplementemia, and dsDNA antibodies in systemic lupus erythematosus. J Rheumatol. 1991; 18: 230-4.

8. Vitali C, Bombardieri S, Jonsson R, Moutsopoulos HM, Alexander EL, Carsons SE, et al. European Study Group on Classification Criteria for Sjögren's Syndrome. Classification criteria for Sjögren's syndrome: a revised version of the European criteria proposed by the American-European Consensus Group. Ann Rheum Dis. 2002; 61: 554-8.

9. Mok CC, Ho LY, Leung HW, Wong LG. Performance of anti-C1q, antinucleosome, and 
anti-dsDNA antibodies for detecting concurrent disease activity of systemic lupus erythematosus. Transl Res. 2010; 156: 320-5.

10. Nasr SH, D'Agati VD, Park HR, Sterman PL, Goyzueta JD, Dressler RM, et al. Necrotizing and crescentic lupus nephritis with antineutrophil cytoplasmic antibody seropositivity. Clin J Am Soc Nephrol. 2008; 3: 682-90.

11. Min DJ, Kim SJ, Park SH, Seo YI, Kang HJ, Kim WU, et al. Anti-nucleosome antibody: significance in lupus patients lacking anti-double-stranded DNA antibody. Clin Exp Rheumatol. 2002; 20: 13-8.

12. Swaak AJ, Aarden LA, Statius van Eps LW, Feltkamp TE. Anti-dsDNA and complement profiles as prognostic guides in systemic lupus erythematosus. Arthritis Rheum 1979; 22: 226-35.

13. Sandhu G, Bansal A, Ranade A, Aggarwal R, Narayanswami G, Jones J, et al. Negative double stranded DNA and anti-Smith antibodies in lupus nephritis. Nephrol Rev. 2012; 4: e13. 